\title{
ANÁLISIS DE LA INFORMACIÓN EN LA PREVENCIÓN DEL CONSUMO DE DROGAS Y OTRAS ADICCIONES
}

\section{INFORMATION ANALISYS IN DRUG CONSUMPTION AND OTHER ADDICTIONS PREVENTION}

\author{
${ }^{1}$ Jose A. García del Castillo, ${ }^{2}$ Carmen López-Sánchez, ${ }^{1}$ Álvaro García del Castillo-López y ${ }^{3}$ Paulo C. Dias \\ ${ }^{1}$ Universidad Miguel Hernández \\ ${ }^{2}$ Universidad de Alicante \\ ${ }^{3}$ Universidad Católica de Braga
}

\section{Abstract}

Traditionally a large proportion of the preventive programs focused in drugs and other addictions among adolescents and young people have drawn on the information as a core strategy to discourage the consumption, using arguments based in the consequences of the deterioration being substance consumers, as well as in the abuse of other non chemical addictive behaviors. The classical approach is based on the rational decision process among young people to do the consumption or not, therefore if they were properly informed about the risks they would choose the no consumption. Is essential to address the importance of three topics around the information issue: degree and quality information among young people, degree and quality information among parents and information source search about drugs and its credibility degree. The models of transmission and information content used by the commercial advertising may be more appropriate and effective than the 'fear resort' within health behaviors and addictions in general. The trend of health message promoters continue to be the communication of risk and negative consequences, following the fear resort way. We propose that would be more effective using positive messages enforcing a healthy lifestyle.

Keywords: Addiction prevention, Addiction information, Fear resort.

\section{Resumen}

Tradicionalmente gran parte de los programas preventivos de drogas y otras adicciones dirigidos a preadolescentes y adolescentes, se han apoyado en la información como estrategia fundamental para disuadir el consumo, utilizando argumentos basados en las consecuencias del deterioro al ser consumidores de sustancias, así como en el abuso de otras conductas adictivas no químicas. El enfoque clásico se apoya en que los jóvenes toman sus decisiones de consumir, o no, en base a una elaboración racional, por tanto en el caso de estar informados de los riesgos que asumen al consumir optarían por no hacerlo. Es fundamental incidir en la importancia que adquieren tres cuestiones alrededor de la información: el nivel y calidad de la información que tienen los jóvenes, el nivel y calidad de la información que tienen los padres y la búsqueda de las fuentes de información sobre drogas y su nivel de credibilidad. Posiblemente los modelos de transmisión y contenido de información que utiliza la publicidad comercial podrían ser más adecuados y eficaces que los del recurso al miedo en el ámbito de los comportamientos de salud, y por supuesto, de las adicciones en general. La tendencia de los promotores de mensajes de salud sigue siendo la de transmitir las consecuencias y los riesgos, en la línea de la apelación al miedo. Presumimos que se alcanzaría una mayor eficiencia con mensajes positivos, reforzando los estilos de vida saludables.

Palabras clave: Prevención de adicciones, Información sobre adicciones, Recurso al miedo. 
La información en sí misma cuenta con muchas formas de ser abordada, entendida y utilizada, hasta el punto de que esas cuestiones serán las que marquen realmente su valor futuro en el conocimiento y en el comportamiento. En cualquier proceso de comunicación humana la transmisión de información es, además de imprescindible, un referente para que se pueda generar una relación de influencia $y$, por lo tanto, de posible cambio. Como indicó Hewstone (1990), la comunicación implica intercambio de mensajes entre dos o más participantes caracterizado por el uso intencional, consciente (a cierto nivel de conciencia) de un sistema simbólico mutuamente inteligible. No existe controversia alguna en la afirmación clásica de que la "información es poder", porque desde el punto de vista global, se entiende que la información es necesaria, o mejor, imprescindible, para el buen desarrollo de cualquier actividad, pero además incide directamente en las posibles formas de influencia por parte de quien la posea.

Según señala Soengas (2009), la información es un bien público y debería de ser accesible por igual para todos los ciudadanos. Este punto de vista incide sobre todo en cuestiones que pueden ser relevantes para el bienestar y la calidad de vida, además de para lo que el autor refiere más expresamente, la sustentación del poder.

La información se construye en su origen con datos, pero al final va mucho más allá construyendo el conocimiento (Rendón, 2005). De esta construcción podríamos considerar el posterior desarrollo del comportamiento, si solamente se sustentara en información y conocimiento, pero sabemos que la forma en la que conducimos nuestras acciones se sustenta además en otros procesos básicos como la motivación, las actitudes y creencias o las emociones, por considerar algunos de ellos.

Una de las cuestiones más importantes acerca de la información la encontramos alrededor de las fuentes. Como veremos la fuente de información pasará a un primer plano, ya que será la garantía de ser más o menos fidedigna, creíble y, por tanto, utilizable. En la actualidad contamos con una amplia diversidad de fuentes gracias a las nuevas tecnologías de la información y la comunicación, lo que por una parte facilita las búsquedas y por otra complica las garantías de autenticidad. De hecho no es difícil encontrar información basada en fuentes terciarias, que tras un largo recorrido se han deteriorado y entran en contradicción o tergiversan a las fuentes primarias.

En la prevención del consumo y en las intervenciones de prevención de adicciones en general, la información es una de las constantes que podemos encontrar en cualquiera de los programas que revisemos en el ámbito nacional e internacional (Hernández, Espada, Piqueras, Orgiles, García, 2013; Rodríguez, Díaz, Gracia, Guerrero, Gómez-Maqueo, 2011; Ringwalt, Clark, Hanley, Shamblen,
Flewelling, 2010). No obstante, una de las cuestiones omnipresentes, en torno al suministro de información, sobre los peligros del consumo de drogas y otras adicciones, es lo controvertido de su impacto en los receptores y cómo puede traducirse en una medida contraproducente, muy al contrario de la intención primaria. En esta línea, textos tan clásicos como el de Bermann (1926), nos ilustran magistralmente del fracaso de medidas informativas para un problema tan crítico: "¿Por qué no obrar por la exhortación al buen sentido, a la razón, mediante carteles de propaganda, conferencias, buenos consejos? Estos medios han fallado lastimosamente. Para mal tan antiguo y tan hondo quedan sin eficacia medidas tan suaves" (Bermann, 1926, 335).

Cuando la información se emplea como única medida preventiva, se relaciona directamente con la percepción de riesgo del receptor. Para que sea realmente preventiva la información debería de incidir sobre el riesgo percibido, cosa que sucede en función de la edad de las personas. Sabemos, en general, que a mayor edad mayor percepción de riesgo y viceversa, independientemente de la cantidad de información que se posea. No obstante, como sugieren algunos autores (García del Castillo, 2012; García del Castillo, García del Castillo-López, Días, López-Sánchez, Gázquez, Lloret y Segura, 2014), el control del riesgo puede estar supeditado a la cantidad de experiencias e información que tenga la persona, aunque muchos comportamientos, aun teniendo información fidedigna, superan la barrera de riesgo asumible, porque entran en juego factores como el azar, la ilusión de invulnerabilidad o la minimización del daño que se podría padecer en el caso de que las cosas no salieran como se habían previsto previamente en base a la información o experiencia.

Las personas, en gran cantidad de ocasiones, son incapaces de hacer valoraciones de las situaciones de una forma totalmente objetiva, entre otros motivos porque pueden estar bajo la influencia directa o indirecta de variables externas a ellos, como por ejemplo los amigos o el propio contexto, o bien, porque las valoraciones que llevan a cabo las hacen de forma subjetiva, disminuyendo la posible vulnerabilidad ante las consecuencias. Las cogniciones pueden perfectamente sesgarse en función de la información recibida (Concha, Bilbao, Gallardo, Páez y Fresno, 2012) y, añadiríamos, de la interpretación que se haga de esa información.

Tradicionalmente gran parte de los programas preventivos de drogas y otras adicciones dirigidos a preadolescentes y adolescentes, se han apoyado en la información como estrategia fundamental para disuadir el consumo, utilizando argumentos en base a las consecuencias de deterioro al ser consumidores. El enfoque clásico se apoya en que los jóvenes toman sus decisiones 
de consumir, o no, en base a una elaboración racional, por tanto en el caso de estar informados de los riesgos que asumen al consumir optarían por no hacerlo. Los estudios clásicos sobre este supuesto muestran la ineficacia de esta medida (Botwin, 1995; Botwin y Botwin, 1992; BangertDrowns, 1988; Bruvold y Rundall, 1988; Tobler, 1986). En un meta-análisis llevado a cabo por Espada, Rosa y Méndez (2003), se verificó nuevamente que los programas basados en la información son menos eficaces que los que utilizan otras estrategias. Pero un dato importante de este estudio es que se verifica que todos los programas preventivos analizados, un total de 15, cuentan con la información como variable interviniente.

El tratamiento que configura el mensaje informativo va a ser crucial para que el impacto en el receptor se materialice de una manera positiva, negativa o neutra. Como veremos, las formas de elaborar el mensaje han sido ampliamente estudiadas desde la psicología y la publicidad para poder influir en los receptores o público objetivo y de este modo inducir a la compra de un producto o servicio. Como señalan Suárez y Pérez $(2001,10)$ "el fin de la publicidad es siempre convencernos y persuadirnos de algo. De alguna manera, debe inducirnos a consumir un producto, a comprar un servicio o a cambiar de opinión respecto a algún tema".

El fin último de los mensajes de salud estaría en esta misma línea de acción, intentando persuadir al receptor de los beneficios que reporta un comportamiento sano frente a uno de riesgo, aunque en muchas ocasiones, como veremos, la búsqueda de información se encamina hacia la intención de consumo, más que a la protección de la salud.

\section{NIVEL DE INFORMACIÓN Y SU INCIDENCIA EN EL CONSUMO DE LOS JÓVENES}

En general existe una gran disparidad de medios por los que la población tiene acceso a la información sobre drogas y otras adicciones. Según el estudio EDADES (PNsD, 2011), las vías por las que más información reciben los ciudadanos españoles, es a través de los medios de comunicación (64,4\%), seguido de los amigos o compañeros de trabajo (24,6\%). Como podemos comprobar en la Figura 1, resulta sorprendente que los profesionales sanitarios únicamente alcancen un $8 \%$ del total o que los cursos especializados no lleguen al $13 \%$.

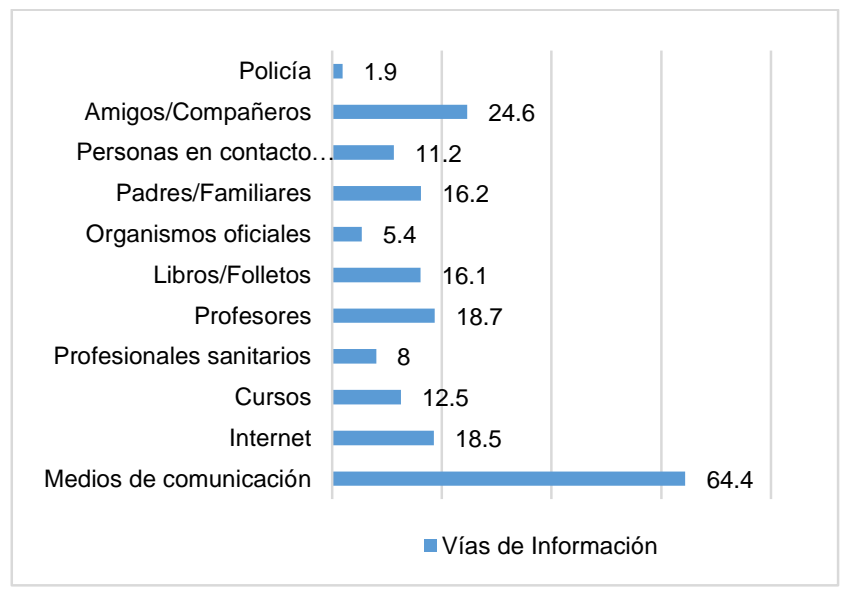

Figura 1. Vías de entrada de la información en población general española (Modificado de EDADES, PNsD, 2011)

Para los más jóvenes, la entrada de información va unida a los programas de prevención que contienen cursos o charlas informativas en su mayor porcentaje (67.5\%). No obstante también cuentan con otras vías de acceso a la información sobre drogas y otras adicciones vinculadas a las madres $(67,3)$, los profesores $(61,4 \%)$ o los medios de comunicación (61\%) (PNsD, 2010). En la Figura 2, podemos comprobar todas las fuentes de información de los jóvenes españoles entre 14 y 18 años de edad.

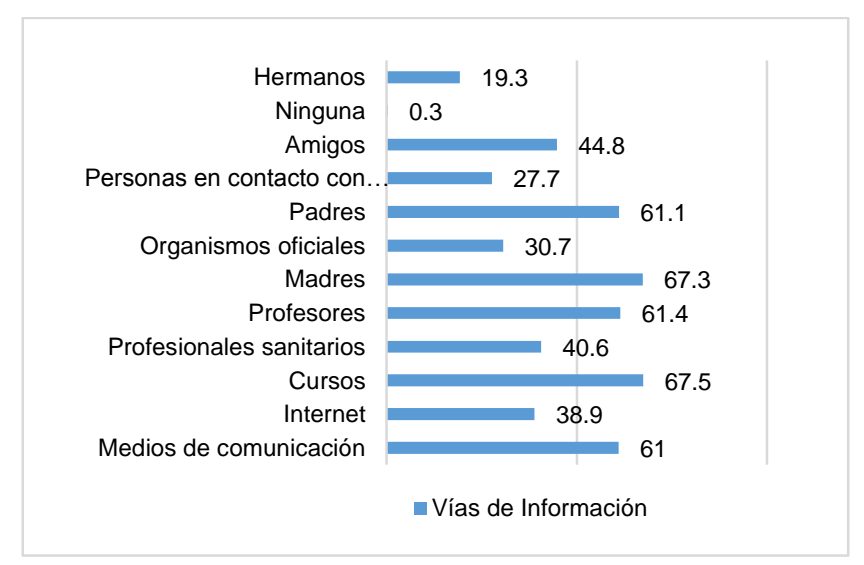

Figura 2. Fuentes de información sobre drogas de jóvenes entre 14-18 años (Modificado de PNsD, 2010)

Es fundamental incidir en la importancia que adquieren tres cuestiones alrededor de la información:

1. El nivel y calidad de la información que tienen los jóvenes.

2. El nivel y calidad de la información que tienen los padres. 
3. La búsqueda de las fuentes de información sobre drogas y otras adicciones, y su nivel de credibilidad.

1. En relación a la primera de estas cuestiones, existe una gran variabilidad en función de la edad de los jóvenes, ya que a mayor edad cuentan con una mayor cantidad de conocimientos, que se pueden atribuir tanto a los programas y acciones preventivas, como a la búsqueda que los propios jóvenes realizan, apuntando hacia un efecto maduración (Gázquez, García del Castillo y Espada, 2011). De hecho en la población universitaria encontramos que los niveles y calidad de la información son más que suficientes tanto en el plano objetivo (López, Santín, Torrico y Rodríguez, 2003) como en el subjetivo o percibido (García del Castillo, Gázquez y García del Castillo-López, 2011) (Figura 3), en comparación con población más joven donde la información se encuentran en niveles medio-bajos (García del Castillo, López-Sánchez, Fernández y Santos 2001), lo que corrobora por una parte el efecto maduración $y$, por otra, los efectos que pueden producir la información preventiva y la búsqueda de información de los propios jóvenes.

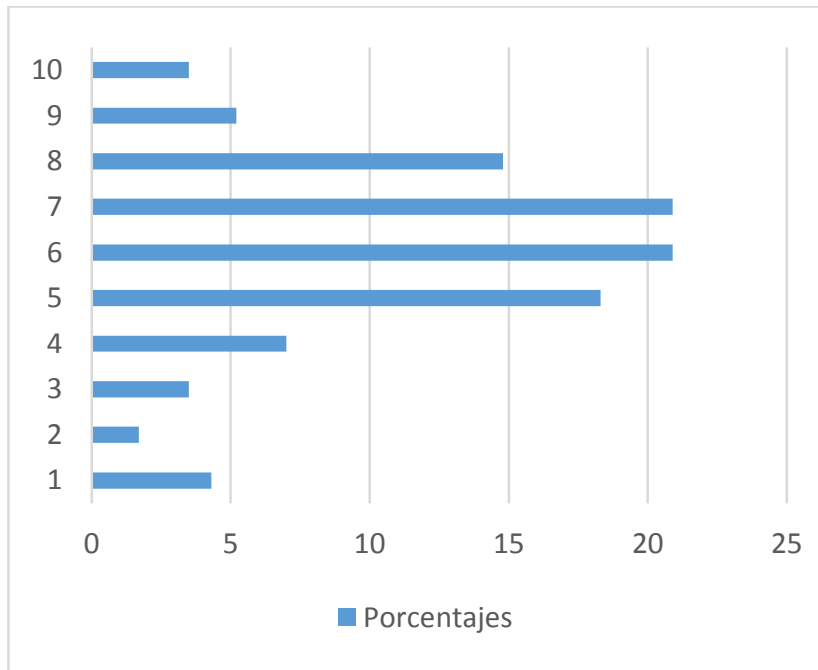

Figura 3. Nivel de información percibida de una población de jóvenes universitarios (Modificado de García del Castillo et al., 2011)

2. El nivel y calidad de la información que tienen los padres ha de ir acompasado con su percepción de responsabilidad en la educación. Más del 75\% de los padres se consideran los máximos responsables de la educación de los hijos (Abbate, 2006; Escutia, Robles, Oudhof, Villafaña y Garay, 2014; García del Castillo et al., 2001; Peréz-Díaz, Rodríguez y Sánchez, 2001). Para ello tienen que estar preparados en un campo de acción que abarca numerosas frentes. Para poder afrontar con éxito el proceso educativo, necesitan contar con un aliado inexcusable que es la información, pero cualquier información no es útil, tiene que ser una información de calidad que les posibilite su tarea y que optimice el proceso educativo. Algunos estudios han recogido la cantidad de información de los padres, bien de forma percibida: "La información que tiene sobre las drogas la considera" (Pinazo y Pons, 2005, 337) (Figura 4), bien de forma cuantitativa (García del Castillo et al., 2001) (Figura 5).

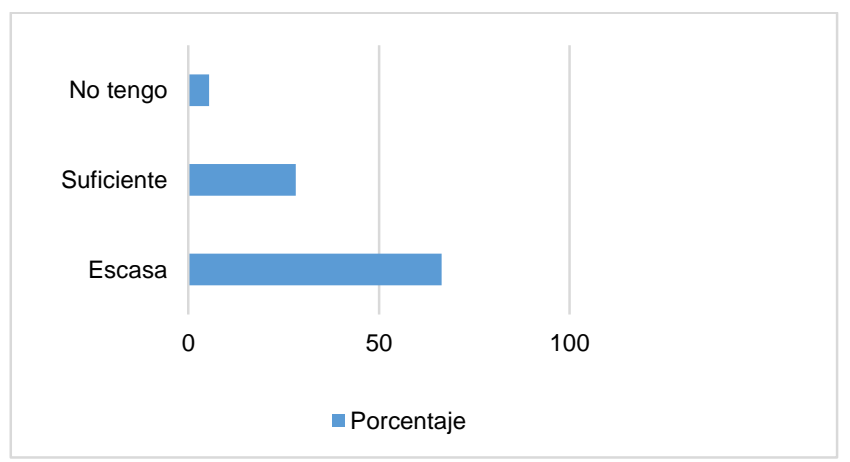

Figura 4. La información percibida sobre drogas (Pinazo y Pons, 2005)

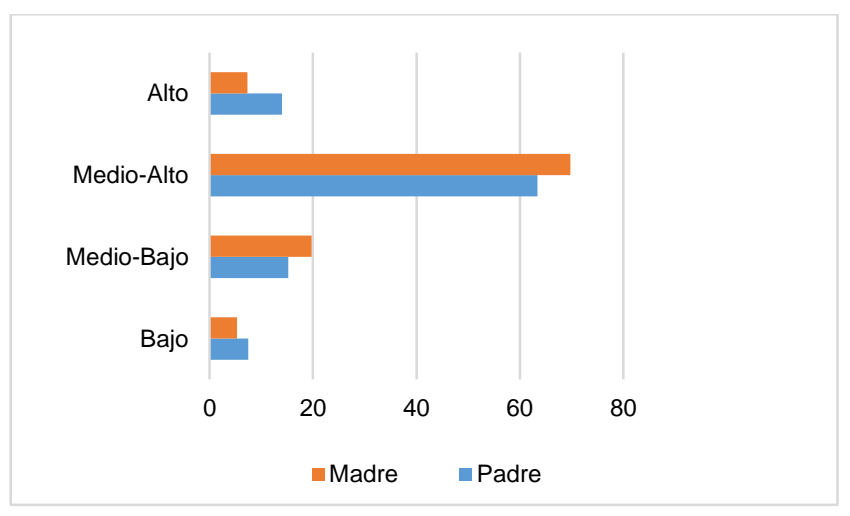

Figura 5. Información cuantitativa sobre drogas de los padres (García del Castillo et al., 2001)

Aunque sea grosso modo, $y$ en secuencias $e$ investigaciones diferentes, coincide que la información percibida es más baja comparada con la real en cuanto a la cantidad y calidad de la información básica que tienen los padres sobre drogas.

3. En cuanto al tercer aspecto, la búsqueda de las fuentes de información sobre drogas y su nivel de credibilidad, habría que señalar en primer lugar que la 
búsqueda de información sobre drogas y otras adicciones por la población universitaria tiene un doble objetivo, por una parte aumentar el nivel de conocimiento $y$, por otra, la intención de consumo de los que recaban la información (Ying, Jisoo, Lewis y Martínez, 2014).

Las sustancias más consultadas se circunscriben al tabaco y el alcohol (drogas legales) y al cannabis y la cocaína (drogas ilegales) (Segura, García del Castillo y López-Sánchez, 2010; García del Castillo et al., 2011). La elección de las fuentes se concentra en más del $73 \%$ de los casos en páginas web de instituciones especializadas y páginas web especializadas, seguidas de fundaciones en un $13 \%$. La mayor credibilidad se encuentra en libros y revistas de divulgación científica seguido de prensa y radio (García del Castillo et al., 2011) (Figura 6).

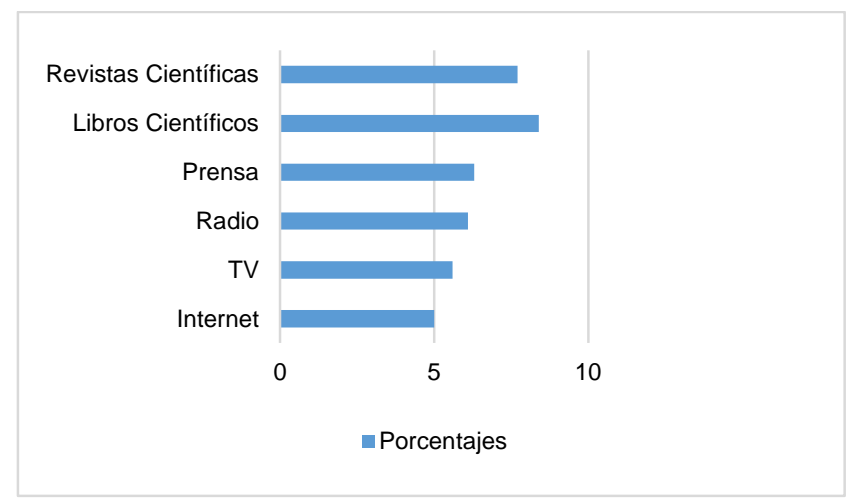

Figura 6. Credibilidad de las fuentes de información sobre drogas (Modificado de García del Castillo et al., 2011)

\section{EL VALOR DE LA INFORMACIÓN SOBRE DROGAS Y OTRAS ADICCIONES Y TIPOS DE MENSAJES}

La información sobre drogas y otras adicciones adquiere un papel preponderante, como hemos señalado, en prácticamente todas las acciones preventivas. Su relación con variables fundamentales en el comportamiento de consumo es incuestionable $y$, de hecho, está implicada en algunas de las variables más significativas vinculadas a la iniciación al consumo, como es la percepción de riesgo y la percepción de vulnerabilidad, dos constructos íntimamente unidos.

Seguimos en la creencia de que la percepción de riesgo regula la probabilidad de comenzar a consumir y de mantener los consumos (García del Castillo, 2012). Los jóvenes desarrollan la capacidad de compensar la información para comenzar a consumir, mediante la ilusión de invulnerabilidad, que los lleva a pensar que las consecuencias negativas del consumo de sustancias se darán en los demás pero no en uno mismo (Mietzel, 2005). Teóricamente, para que las variables de percepción de riesgo y percepción de vulnerabilidad funcionen adecuadamente, se ha de dar la condición de que el joven cuente con información de calidad, que asuma como peligroso el comportamiento de consumo en sí mismo y se diluya la ilusión de invulnerabilidad. La percepción de vulnerabilidad, según Gil-Lacruz (2007) potencia la motivación para llevar un estilo de vida sano.

Para que los niveles de información sobre sustancias se empiecen a configurar, los jóvenes tienen que alcanzar una edad entre los 9 o 10 años, que podríamos considerar temprana (Sierra, Pérez, Pérez y Núñez, 2005). Como señalan estos autores, los padres abordan el tema de las drogas y otras adicciones a partir de las preguntas de sus hijos, utilizando mensajes de miedo fundamentalmente ("son malísimas, te destrozan la vida, te enganchan,..."), que suelen resultar bastante confusos para los receptores jóvenes, sobre todo, si tienen modelos cercanos que consumen sustancias socialmente aceptadas o comportamientos de adicción psicológica. Por otra parte sabemos que los primeros consumos de sustancias legales, como por ejemplo el alcohol, suelen inducirse por los propios progenitores de manera indirecta, permitiendo que sus hijos beban a colación de algún acontecimiento familiar festivo o recreativo y de manera directa como modelos consumidores. Según Espada, Pereira y García-Fernández (2008), uno de los indicadores principales en el consumo de alcohol de los jóvenes está asociado con el consumo de su mejor amigo y de sus hermanos.

La información persuasiva, desde los estudios clásicos de la Psicología Social, se apoya en las investigaciones del grupo de Yale sobre persuasión (Hovland, Janis y Kelley, 1953; Hovland y Janis, 1959). Como recoge (Moya, 1999), la repercusión de los mensajes en los receptores, según las teorías de la persuasión, se modula en cuatro procesos:

- Atención: no todos los mensajes son atendidos por el receptor.

- Comprensión: los mensajes ambiguos, complejos o dispersos pueden perder su efecto.

- Aceptación: cuando los receptores, después de superar los dos procesos anteriores, están de acuerdo con el mensaje recibido.

- Retención: para que la información sea realmente persuasiva, necesita perdurar en el tiempo.

Posteriormente será McGuire (1969, 1985), quien proponga reducir los procesos a dos: recepción y aceptación. Con el desarrollo de la teoría de la respuesta cognitiva, se da un nuevo paso en los procesos del mensaje persuasivo, iniciado ya por McGuire. Esta teoría formula que los mensajes recibidos por el receptor son evaluados con 
los conocimientos y actitudes que previamente tiene el receptor para que puedan generar respuestas cognitivas. Como señalan (Petty y Cacioppo, 1981, 1986; Petty, Ostrom y Brock, 1981), aquellos pensamientos que coinciden con el mensaje tienen efecto persuasivo, pero los que no coinciden no generan persuasión e incluso pueden actuar de forma inversa (el llamado "efecto boomerang"). Investigaciones posteriores (Briñol, De la Corte y Becerra, 2001) demuestra que cuando los mensajes son más convincentes y coinciden con que el estado de ánimo de la persona es positivo, la probabilidad de que se generen pensamientos positivos hacia los mensajes persuasivos es mayor. Estos mismos investigadores han propuesto posteriormente que la autoafirmación aumenta la persuasión cuando los pensamientos del receptor sobre el mensaje recibido son favorables y viceversa (Briñol, Becerra, Gallardo, Horcajo y Valle, 2004).

Tradicionalmente los mensajes se plantean en dos formatos claramente diferenciados, de hecho Aristóteles ya lo establecía así en su "Retórica", por una parte, los que se basan en lo puramente racional, es decir, que tratan de plantear la información lo más realistamente posible, intentando cubrir objetivos claros y concisos haciendo siempre referencia a estudios contrastados y fehacientes. Como señalan Briñol, De la Corte y Becerra (2001), los mensajes racionales intentan argumentar basándose en la deducción o en la inferencia para forzar en el receptor el análisis y la reflexión. Y por otra parte, los mensajes emocionales, que pueden tener una doble vertiente:

- Emocional positiva, que es la que suele emplearse mayoritariamente en los mensajes publicitarios y giran en torno a emociones de las percibidas como "buenas" o positivas (amor, felicidad, alegría, gozo, afecto, etc.).

- Emocional negativa, lo que denominamos apelación o recurso al miedo, que se sustenta en mensajes que intentan activar la emoción de miedo ante posibles comportamientos.

Según Briñol et al. (2001) existe evidencia empírica de la eficacia de los mensajes con mezcla de racional y emotivo, aunque en la actualidad sigue la controversia cuando se busca efectividad en el cambio de actitudes del receptor, de tal forma que los mensajes con componentes racionales serán mejores cuando el componente que se desea modificar es el cognitivo. En cambio cuando el que se desea modificar es el componente afectivo, tendrán más efectividad los mensajes emotivos. No obstante parece ser que existe evidencia empírica en los dos sentidos, es decir, que autores como Millar y Millar (1990), señalan que los argumentos serían al contrario, un mensaje racional sería más efectivo para el cambio del componente afectivo y un mensaje emocional para el cambio del componente cognitivo.

En el marketing de salud resulta complicado no apelar al miedo en sus mensajes, porque normalmente la información se dirige hacia las consecuencias de un comportamiento de riesgo. Ordoñana y Gómez (2002), apuntan que los mensajes preventivos y de promoción de la salud pueden generar efectos tanto positivos como negativos, en función de las características del mensaje.

El estudio de los mensajes de miedo cuenta con propuestas teóricas consistentes, como el Modelo de Respuesta Paralela Extendido que se desarrolla a partir de los formulados por Leventhal $(1970,1971)$ sobre mensajes de miedo y Rogers $(1975,1983,1985)$ desde la teoría de la Motivación Protectora. Pero es Witte (1992a, 1992b, 1998; Witte y Allen, 2000), quien plantea que depende de cómo se presente el mensaje de miedo para que la persona lo valore como amenaza en función de la severidad percibida sumada a la cantidad de vulnerabilidad que perciba. Si el mensaje de miedo lo interpreta el sujeto como superable no se producirá el proceso motivacional deseado.

La cuestión principal de este tipo de mensajes, siempre ha girado en torno a la cantidad de efectividad que pueden tener. Parece ser que los mensajes que suscitan miedo son más efectivos cuando se dan las siguientes condiciones (Rogers y Mewborn, 1976):

- $\quad$ Extrema gravedad: el mensaje proporciona sólidos argumentos sobre la posibilidad de que el receptor sufra de forma extrema alguna consecuencia negativa.

- Posibilidad de ocurrencia: los argumentos explican que estas consecuencias negativas son muy probables que ocurran si no se adoptan las recomendaciones sugeridas.

- Evitación del problema: el mensaje asegura que con la adopción de sus recomendaciones evitará con seguridad las consecuencias negativas.

- Autoeficacia: creencia del receptor del mensaje de que podrán llevar a cabo las recomendaciones sin muchas dificultades.

En esta misma línea Ordoñana y Gómez (2002), afirman que para que un mensaje de miedo tenga una mayor probabilidad de generar efectos positivos en el comportamiento del sujeto, deben de cumplirse algunas condiciones: 
a) Tienen que activar una respuesta atencional, sustentada en argumentos con una consistencia óptima para el sujeto receptor.

b) Tiene que contar con consejos que el sujeto perciba claramente que le evitarán cualquier tipo de riesgo en el caso de seguirlos.

c) Se tiene que utilizar información verídica y creíble.

Las recomendaciones para que aumente la eficacia de los mensajes de miedo, según Rodríguez (2011), atenderían a los siguientes supuestos:

- $\quad$ Evaluar detenidamente los riesgos reales.

- Tener conocimiento de los destinatarios para poder adecuar los mensajes a la audiencia.

- La advertencia debe de experimentarse como cercana, dentro de unos márgenes que puedan repercutir en el receptor objetivo.

- La fuente del mensaje debe de ser totalmente creíble.

- Enviar los mensajes pautados para recordar constantemente la situación.

- Como en los casos anteriores, las recomendaciones o consejos deben de presentarse con claridad para que no exista ninguna duda de que si se siguen se evitará el daño.

Aunque las investigaciones han ido avanzando en la búsqueda de soluciones para resolver la controversia sobre la efectividad de los mensajes de miedo, la utilización de este tipo de información sigue siendo una constante en un gran número de diseños, fundamentalmente los referidos a temas de salud y campañas de tráfico.

\section{CONSIDERACIONES FINALES}

Posiblemente los modelos de transmisión y contenido de información que utiliza la publicidad comercial podrían ser más adecuados y eficaces que los del recurso al miedo en el ámbito de los comportamientos de salud. La tendencia de los promotores de mensajes de salud sigue siendo la de transmitir las consecuencias y los riesgos, en la línea de la apelación al miedo. Presumimos que se alcanzaría una mayor eficiencia con mensajes positivos, reforzando los estilos de vida saludables.

Al igual que las campañas publicitarias comerciales que se apoyan en las bases de la influencia para promocionar sus productos, se podría proponer adaptar los principios de influencia de Cialdini (1984) para aplicarlos a los programas de prevención:

1. Reciprocidad: Las personas tienen necesidad de devolver cuando reciben algo, para poder reestablecer el equilibrio. Un ejemplo en el ámbito de las adicciones sería el de potenciar la figura de los mediadores de salud que ofrecen su ayuda a los jóvenes a cambio de que modifiquen su estilo de vida.

2. Coherencia y compromiso: Las personas tienen necesidad de comportarse consecuentemente con lo que piensan, hacen y dicen. Un ejemplo en el ámbito de las adicciones sería modificar las actitudes de los jóvenes hacia el mantenimiento de la salud.

3. Aprobación social: Las personas tienden a imitar el comportamiento de los demás para no equivocarse. Un ejemplo en el ámbito de las adicciones sería promocionar los comportamientos saludables que ostentan la mayoría de los jóvenes, para que pasaran a ser un comportamiento normativo.

4. Simpatía: Las personas son mucho más influenciables por modelos simpáticos y agradables. Un ejemplo en el ámbito de las adicciones sería implementar campañas con comportamientos positivos, utilizando modelos atractivos que sirvan de referencia a la población joven.

5. Autoridad: Las personas tienden a dar más credibilidad y ser más obedientes ante quien ostenta autoridad. Un ejemplo en el ámbito de las adicciones sería apoyar campañas de salud mediante referentes de autoridad.

6. Escasez: Las personas tienden a darle más valor a las cosas que escasean. Un ejemplo en el ámbito de las adicciones sería "prohibir" determinados comportamientos saludables para incitar el interés de los jóvenes.

Las acciones preventivas que se apoyan en la información como soporte prioritario, siguen utilizando un diseño orientado a la descripción de las sustancias y a las consecuencias del consumo, al igual que los comportamientos de adicción psicológica. Lemas como "Drogas: más información, menos riesgos", no son coherentes con la realidad preventiva, al igual que tampoco lo fueron los mensajes de "Dí no a las drogas" criticado ampliamente por Newcomb y Bentler (1988). Habría que intentar ser consecuentes con la investigación y suministrar la información sobre drogas y otras adicciones desde un campo más amplio de acción, remarcando los aspectos positivos de los estilos de vida saludables, que puedan servir de referente normativo a la población joven. 


\section{REFERENCIAS}

Abbate, F. (2006). Educación sexual del adolescente. Rev. Asociación Médica Argentina, 119(3), 12-4

Bangert-Drowns, R.L. (1988). The effects of school-based substance education: A meta-analysis. Journal of Drug Education, 18, 243-265.

Bermann, G. (1929). Toxicomanías. Buenos Aires: El Ateneo.

Botwin, G.J. (1995). Entrenamiento en habilidades para la vida y prevención del consumo de drogas en adolescentes: consideraciones teóricas y hallazgos empíricos. Psicología Conductual, 3(3), 333-356.

Botwin, G.J. y Botwin, E.M. (1992). Adolescent tobacco, alcohol, and drug abuse: Prevention strategies, empirical findings, and assessment issues. Development and Behavioral Pediatrics, 13, 290-301.

Briñol, P., Becerra, A., Gallardo, I., Horcajo, J. y Valle, C. (2004). Validación del pensamiento y persuasion. Psicothema, 16(4), 606-610.

Briñol, P., De la Corte, L. y Becerra, A. (2001). Qué es persuasión. Madrid: Biblioteca Nueva.

Bruvold, W.H. y Rundall, T.G. (1988). A meta-analysis and theoretical review of school based tobacco and alcohol intervention programs. Psychology and Health, 2(1), 53-78.

Cialdini, R.B. (1984). Influence: The Psychology of Persuasion. New York: Morrow.

Concha, D., Bilbao, M.A., Gallardo, I., Páez, D. y Fresno, A. (2012). Sesgos cognitivos y su relación con el bienestar subjetivo. Salud y Sociedad, 3 (2), 115-129.

Escutia, M., Robles, E., Oudhof, H., Villafaña, L.G. y Garay, J. (2014). Tareas de crianza de padres mexicanos con hijos adolescents. Ciencia Ergo Sum [en linea] 2014, 21 (Marzo-Junio): [Fecha de consulta: 8 de julio de 2014] Disponible en:<http://www.redalyc.org/articulo.oa?id=10429976003> ISSN 1405-0269.

Espada, J.P., Pereira, J.R. y García-Fernández, J.M. (2008). Influencia de los modelos sociales en el consumo de alcohol de los adolescents. Psicothema, 20(4), 531-537.

Espada, J.P., Rosa, A.I. y Méndez, F.X. (2003). Eficacia de los programas de prevención escolar de drogas con metodología interactiva. Health \& Addictions/Salud y Drogas, 3(2), 61-81.

García del Castillo, J.A. (2012). Concepto de percepción de riesgo y su repercusión en las adicciones. Health \& Addictions/Salud y Drogas, 12(2), 133-151.

García del Castillo, J.A., García del Castillo-López, A, Días, P., López-Sánchez, C., Gázquez, M., Lloret, D. y Segura, M.C. (2014). Percepción de riesgo hacia el consumo de alcohol. En J.A. García del Castillo y P. Días (coord.). Estudos sobre o álcool: Contributos para a prática. Lisboa: PEDAGO (En prensa).

García del Castillo, J.A., López-Sánchez, C., Fernández, S. y Santos, C. (2001). Estudio sobre tabaco, alcohol y otras drogas en El Campello. Alicante: INID.

Gázquez, M., García del Castillo, J.A. y Espada, J.P. (2011). Eficacia diferencial de dos programas de prevención escolar sobre el consumo de tabaco, según el tipo de aplicador. Psicothema, 23(4), 537-543.
Gil-Lacruz, M. (2007). Psicología social: Un compromiso aplicado a la salud. Zaragoza: Universidad de Zaragoza.

Hernández, O., Espada, J.P., Piqueras, J.A., Orgiles, M. y García, J.M. (2013). Programa de prevención del consumo de drogas SALUDA: evaluación de una nueva versión en adolescentes españoles. Health \& Addictions/Salud y Drogas, 13(2), 135-144

Hewstone, M. (1990). The 'ultimate attribution error'? A review of the literature on intergroup causal attribution. European Journal of Social Psychology, 20(4), 311-335.

Hovland, C. I. y Janis, I. L. (1959). Personality and persuasibility. New Haven, CT: Yale University Press.

Hovland, C.I., Janis, I. y Kelley, H.H. (1953). Comunication and persuasion. New Haven, CT: Yale University Press.

Leventhal, H. (1970). Findings and Theory in the Study of Fear Communications. In L. Berkowitz (Ed.). Advances in Experimental Social Psychology (119-186). California: Academic Press.

Leventhal, H. (1971). Fear appeals and persuasion: The differentiation of a motivational construct. American Journal of Public Health, 61, 12081224.

López, M.J., Santín, C., Torrico, E. y Rodríguez, J.M. (2003). Consumo de sustancias psicoactivas en una muestra de jóvenes universitarios. Psicología y Salud, 13(1), 5-17.

McGuire, W. J. (1969). The nature of attitudes and attitude change. In G. Lindzey y E. Aronson (Eds.). The handbook of social psychology. New York: Random.

McGuire, W. J. (1985). Attitudes and attitude change. In G. Lindzey y E. Aronson (Eds.). The handbook of social psychology. New York: Random.

Mietzel, G. (2005). Claves de la psicología evolutiva: Infancia y juventud. Barcelona: Herder.

Millar, M.G. y Millar, K.U. (1990). Attitude change as a function of attitude type and argument type. Journal of Personality and Social Psychology, 59, 217-228.

Moya, M. (1999). Persuasión y cambio de actitudes. En J.F. Morales (coord.). Psicología social. Madrid: McGraw- Hill/Interamericana de España (153-170).

Newcomb, M. D. y Bentler, P. M. (1988). Consequences of adolescent drug use: Impact on the lives of young adults. Beverly Hills, CA: Sage Publications

Ordoñana, J.R. y Gómez, J. (2002). Amenaza y persuasión en mensajes de salud sobre consume de alcohol: de la teoría a la práctica. Adicciones, 14(4), 479-485.

Peréz-Díaz, V., Rodríguez, J.C. y Sánchez, L. (2001). La familia Española ante la educación de sus hijos. Colección de Estudios Sociales, 5. Barcelona: Fundación "La Caixa".

Petty, R. E. y Cacioppo, J.T. (1981). Attitudes and persuasion: Classic and contemporary approaches. Dubuque, Iwoa: Wm. C. Brown (Reprinted 1996, Westview Press, Boulder, CO).

Petty, R.E. y Cacioppo, J.T. (1986). Communication and persuasion: Central and peripheral routes to attitude change. NY: Springer-Verlag.

Petty, R.E., Ostrom, T.M. y Brock, T.C. (1981). Cognitive responses in persuasion. Hillsdale, NJ: Erlbaum. 
Pinazo, S. y Pons, J. (2005). La implicación de los padres en los programas preventivos del consume de drogas: Un estudio empírico. En J.R. Fernández y R. Secades (cords.). Intervención familiar en la prevención de las drogodependencias. Madrid: Plan Nacional sobre Drogas.

PNsD (2010). Encuesta estatal sobre uso de drogas en enseñanzas secundarias (ESTUDES). Madrid: Plan Nacional sobre Drogas. Ministerio de Sanidad, Servicios Sociales e Igualdad. Gobierno de España.

PNsD (2011). Encuesta domiciliaria sobre alcohol y drogas en España (EDADES). Madrid: Plan Nacional sobre Drogas. Ministerio de Sanidad, Servicios Sociales e Igualdad. Gobierno de España.

Rendón, M.A. (2005). Relación entre los conceptos: información, conocimiento y valor. Semejanzas y diferencias. Ciencias de la Información, Brasília, 34(2), 52-61.

Ringwalt, C. L., Clark, H. K., Hanley, S., Shamblen, S. R. y Flewelling, R. L. (2010). The effects of Project ALERT one year past curriculum completion. Prevention Science, 11, 172-184.

Rodríguez, R. (2011). La efectividad del uso del miedo como factor persuasivo en la comunicación de riesgos en las crisis sanitarias. Revista de Comunicación y Salud, 1(2), 33-46.

Rodríguez, S.E., Díaz, D.B., Gracia, S.E., Guerrero, J.A. y Gómez-Maqueo, E.L. (2011). Evaluación de un programa de prevención del consumo de drogas para adolescents. Salud Mental, 34, 27-35.

Rogers, R. W. (1975). A protection motivation theory of fear appeals and attitude change. Journal of Psychology: Interdisciplinary and Applied, 91(1), 93-114.

Rogers, R.W. (1983). Cognitive and physiological processes in fear appeals and attitude change: a revised theory of protection motivation. En J.R. Cacioppo y R.E. Petty (eds.). Social Psychology: a source book (153156). Nueva York: Guildford Press.

Rogers, R.W. (1985). Attitude change and information integration in fear appeals. Psychological Reports, 56(1), 179-182.

Rogers, R.W. y Mewborn, C.R. (1976). Fear appeals and attitudes change: Effects of a threat's noxiousness, probability of occurrence and the efficacy of coping responses. Journal of Personality and Social Psychology, 34, 54-61.

Segura, M.C., García del Castillo, J.A. y López-Sánchez, C. (2010). Patrones de uso y búsqueda de información sobre adicciones en Internet. Health and Addictions/Salud y Drogas, 10(1), 111-135.

Sierra, D.R., Pérez, M., Pérez, A. y Núñez, M. (2005). Representaciones sociales en jóvenes consumidores y no consumidores de sustancias psicoactivas. Adicciones, 17(4), 349-360.

Soengas, X. (2009). Los vínculos entre la información y el poder en la sociedad actual. Revista ICONO 14, 12, 292-322.

Suárez, J.C. y Pérez, M.A. (2001). La publicidad al desnudo. Sevilla: MAD.

Tobler, N. S. (1986). Meta-analysis of 143 adolescent drug prevention programs: quantitative outcome results of program participants compared to a control or comparison group. Journal of Drug Issues, 16(4), 537-567.

Witte, K. (1992a). Message and conceptual confounds in fear appeals: the role of threat, fear, and efficacy. The Southern Communication Journal, 58, 147-155.
Witte, K. (1992b). Putting the fear back into fear appeals: the extended parallel process model. Communication Monographs, 59, 329-349.

Witte, K. (1998). Fear as motivator fear as inhibitor: Using the Extended Parallel Process Model to explain fear appeal successes and failures. In P. Andersen y L. Guerrero (Eds.). Handbook of Communication \& Emotion. (423-450). California: Academic Press.

Witte, K. y Allen, M. (2000). A meta-analysis of fear appeals: implications for effective public health campaigns. Health Education \& Behavior, 27(5), 591-615.

Ying, M.A., Jisoo, A.H.N., Lewis, N. y Martínez, L. (2014). Are drug prevention efforts fulfilling the informational needs of college students? A survey of engagement with information about topics related to marijuana and amphetamines from media, medical and interpersonal sources. 142nd APHA Annual Meeting and Exposition. New Orleans. 